\title{
Laboreal
}

Volume $12 \mathrm{~N}^{\circ} 2$ | 2016

Equipamentos de Proteção Individual II

\section{La actualidad del modelo obrero italiano para la lucha a favor de la salud en el trabajo}

A atualidade do modelo operário italiano nas lutas pela saúde no trabalho L'actualité du modèle ouvrier italien dans les luttes pour la santé au travail aujourd'hui

The relevance of the Italian workers' model in the struggle for health at work today

\section{Laurent Vogel}

\section{OpenEdition}

\section{Journals}

Edición electrónica

URL: http://journals.openedition.org/laboreal/2122

DOI: $10.4000 /$ laboreal. 2122

ISSN: 1646-5237

\section{Editor}

Universidade do Porto

\section{Referencia electrónica}

Laurent Vogel, « La actualidad del modelo obrero italiano para la lucha a favor de la salud en el trabajo », Laboreal [En línea], Volume 12 №2 | 2016, Publicado el 01 diciembre 2016, consultado el 09 octubre 2019. URL : http://journals.openedition.org/laboreal/2122 ; DOI : 10.4000/laboreal.2122

Este documento fue generado automáticamente el 9 octubre 2019.

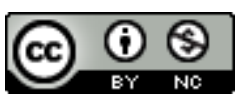

Laboreal está licenciado com uma Licença Creative Commons - Atribuição-NãoComercial 4.0 Internacional. 


\section{La actualidad del modelo obrero italiano para la lucha a favor de la salud en el trabajo}

A atualidade do modelo operário italiano nas lutas pela saúde no trabalho L'actualité du modèle ouvrier italien dans les luttes pour la santé au travail aujourd'hui

The relevance of the Italian workers' model in the struggle for health at work today

\section{Laurent Vogel}

\section{NOTA DEL EDITOR}

http://dx.doi.org/10.15667/laborealxii0216lv

Manuscrito recibido em: abril/2016

Aceptado tras peritage: abril/2016

“Al principio, algunos de mis compañeros de trabajo me parecían estúpidos, no tenían nada que decir. Después, sin embargo, luchando con ellos descubrí que no era así. Eran personas que sí tenían algo que decir, aunque no lo expresaran inmediatamente, tenían un cerebro y sabían utilizarlo. Lográbamos hablar de todo y la política me enseñó a entender a los demás, a valorar a cualquiera, a darme cuenta de que las buenas cosas pueden venir de cualquiera. También aprendí a entenderlas, a no tener prejuicios, a creer que las personas pueden cambiar, madurar, aportar algo. Si alguien no lo hace, quizás se deba a que algo le bloquea, y es esa cosa que hay que eliminar. Eso lo comprendí gracias a la lucha en la fábrica, porque esa era mi vida, pero sin el filtro de la política no sé si hubiera llegado a entenderlo. Eso es lo que quiero recordar de aquella época, porque más allá de las satisfacciones y las victorias momentáneas que el patrón termina por recuperar, eso es lo que queda. Es la prueba de que lo que pasó ha tenido un sentido que sigue intacto 
y válido hasta hoy y que sienta las bases de una nueva temporada, que gracias a ello podrá realizarse un día" (Roberto Sibona, ex obrero de FIAT en Turín entrevistado por Polo, 1989, p. 199).

1 El modelo obrero italiano refiere a una serie de prácticas de lucha a favor de la salud en el trabajo llevadas a cabo en Italia desde principios de los años 60, y que se extendieron a una escala extraordinaria a lo largo de los años 70 . Aunque su denominación refiere a un país específico, también se han desarrollado experiencias comparables fuera de Italia, en un contexto general idéntico marcado por una renovación de la lucha obrera en materia de condiciones de trabajo. En determinadas situaciones la influencia directa del movimiento italiano queda ampliamente demostrada. Es el caso, por ejemplo, en Francia con la lucha de los trabajadores inmigrantes en dos filiales del grupo Peñarroya en 1971 y 1972 donde, a través del colectivo militante Cahiers de mai pudieron utilizarse metodologías de intervención elaboradas en Italia (Pitti, 2009; Pitti, 2010). Asimismo, en Argentina vemos la influencia directa del modelo italiano en la lucha del astillero naval Astarsa al norte de Buenos Aires durante la cual una nueva generación de obreros militantes se enfrentaron tanto a la patronal, como a la burocracia sindical peronista (Lorenz, 2007). En Brasil, la influencia de las experiencias italianas ha jugado un papel importante a finales de los años setenta, en un contexto político en el cual organizaciones sindicales salen progresivamente de la clandestinidad y juegan un papel importante en las luchas contra la dictadura. Las temáticas de salud laboral reciben una atención fuerte por parte de los nuevos protagonistas del sindicalismo con la creación de la Comissão Intersindical de Saúde e Trabalho (Cisat), en 1978 en São Paulo, y dos años más tarde del Departamento Intersindical de Estudos e Pesquisas de Saúde (DIESAT) a nivel nacional (Muniz, Brito, Reis de Souza, Athayde \& Lacomblez, 2013).

2 En otros casos, no hubo necesariamente una influencia directa; se originó más bien un flujo general de ideas en los debates internacionales del movimiento obrero o estudiantil. Estas evoluciones políticas implicaban asimismo conflictos y transformaciones dentro de las principales disciplinas vinculadas con la prevención en los lugares de trabajo. En Francia, por ejemplo, el giro hacia la ergonomía impulsado por el equipo de Alain Wisner del Centro Nacional de Artes y Oficios se produjo a raíz de las movilizaciones sindicales de la CFDT, muy similares a las del modelo obrero italiano (Teiger, Barbaroux, David, Duraffourg, Galisson, Laville \& Thareaut, 2006). Se afirmaba, por vez primera, en esta disciplina una prioridad acordada a la salud de los trabajadores frente a la productividad y llevó a una profunda renovación de las metodologías de intervención.

El presente artículo resume en grandes líneas las características esenciales del modelo obrero italiano. A continuación, cuestiona la actualidad de dicho modelo para la lucha por la salud en el trabajo. La bibliografía permite acceder a obras más amplias y sistemáticas [1] Esperemos que el renacimiento de la historiografía sobre la salud en el trabajo (Bruno, Geerkens, Hatzfeld \& Omnès, 2011) lleve a un programa de investigación comparativa, europeo e internacional sobre esta época fundamental.

\section{¿Por qué Italia?}

4 Podríamos preguntarnos por qué un determinado país se asocia con experiencias que parecen ser la expresión de un desarrollo internacional en el movimiento obrero. En breve, la respuesta guarda relación con cuatro elementos: la continuidad particular de 
la época de lucha que se extiende sobre al menos dos décadas [2]; su intensidad y radicalismo; su capacidad para articularse con la lucha política conjunta que caracterizó la sociedad italiana, y la conceptualización de las experiencias prácticas que permitieron una propagación rápida en todo el territorio nacional y en gran número de empresas de distintos sectores. Esta fuerza singular de las movilizaciones a favor de la salud en el trabajo supuso una de las fuentes de la profunda dinámica anticapitalista que caracterizó la sociedad italiana en los años 60 y 70. También fue el resultado de la misma. Esto le permitió articularse con numerosos otros campos de lucha relacionados con la escuela, la salud, la psiquiatría [3], la vivienda, la sexualidad, etc. El verdadero "milagro italiano" se sitúa allí, más bien que en los elevados porcentajes de crecimiento económico, o la acelerada transición de una sociedad ampliamente rural y conservadora a una sociedad industrial avanzada. Destaca la autonomía de la política y el papel imprescindible de la voluntad organizada de las clases subalternas. Se enmarca en contra de un catastrofismo fatalista que solo vislumbra una ruptura con el capitalismo en situaciones de miseria extrema y de desorganización del Estado vinculada con guerras o una implosión del poder de los de arriba.

5 Cuando se pregunta por la transmisión de la conciencia de los oprimidos a más largo plazo en la historia, se observan en el modelo obrero elementos directamente procedentes de la experiencia de las dos generaciones anteriores. La lucha llevada a cabo por los consejos obreros en Turín en 1919-1920 y la situación insurreccional que caracterizó el triángulo Turín-Milán-Génova en el noroeste de Italia en 1943-1945, aportan referencias que vuelven a surgir hacia finales de los años 60 en la reivindicación de un control obrero sobre las empresas, a partir de la necesidad y la subjetividad obrera como expresión de una conciencia colectiva de clase que se construye sobre la base de las relaciones objetivas de trabajo. Tanto las biografías individuales de algunos protagonistas [4] como el papel de laboratorio colectivo desempeñado por Turín concuerdan con la visión de la historia de Walter Benjamin: los oprimidos tienen la capacidad, a través de su lucha, de avivar las chispas del pasado que creíamos perdidas y apagadas. El modelo obrero saca buena parte de su dinamismo de esta tradición reactivada. Esta transmisión que repara las rupturas de la historia es aún más ejemplar dado que se produjo a pesar de los considerables elementos de discontinuidad tanto a nivel sociológico (inmigración masiva de trabajadores del Mezzogiorno, taylorización de la organización del trabajo), como a nivel político (veinte años de dictadura fascista, estalinización del partido comunista, escisión sindical durante la guerra fría).

6 Carnevale y Baldasseroni (2011) fijan también la atención en las primeras alianzas entre militantes obreros y médicos progresistas a principios del siglo XX con el fin de combatir las enfermedades causadas por exposiciones profesionales. Llevan a investigaciones comunes.

7 Otro hilo conductor aparece de manera menos explícita: una cultura cristiana popular de igualitarismo radical. Se trata de un componente esencial de numerosas luchas en la Italia preindustrial. Las obras históricas de Carlo Ginzburg demostraron que numerosas herejías expresaban una potente cultura subterránea de las clases subalternas. La valoración del "obrero-masa" [5] que refería a los trabajadores no calificados, planteaba el tema del igualitarismo tanto en las fábricas como en el seno de las organizaciones sindicales. Se enfrentaba a menos reticencias en el sindicalismo cristiano (más específicamente en la federación de la metalurgia FIM) que en la FIOM (la federación de 
la metalurgia de la CGIL) que se basaba más en los trabajadores calificados (Galli, 1997). Cabe recordar también la atención prestada continuamente a la creación de instrumentos que liberaran la voz de los trabajadores que desde la escuela primaria habían sido acostumbrados a someterse y callarse. Existen profundas afinidades entre la crítica a la escuela capitalista por los alumnos de Barbiana (Lettre à une maîtresse d'école par les élèves de l'école de Barbiana,1968) y la riqueza del lenguaje hablado y escrito de las creaciones gráficas y hasta teatrales o cinematográficas vinculadas con el modelo obrero. Podemos acercar la teatralidad de las manifestaciones callejeras donde sonaban los tambores, la repentina irrupción de cortejos internos en los talleres rompiendo los horarios ordinarios de la fábrica, la creatividad poética de los eslóganes ("Agnelli, l'Indocina c'è l'hai nell'officina"), los colores recargados de las pancartas con sus múltiples expresiones de fiesta, a la ruptura con lo cotidiano y a la transgresión que va desde el carnaval hasta el sabbat. Asimismo, la reivindicación del papel de la ciencia profana hace eco al cuestionamiento del monopolio de los conocimientos religiosos por los clérigos.

\section{Características esenciales del modelo obrero}

Podríamos intentar resumir las características esenciales del modelo obrero italiano en cuatro puntos. No se trata de una reconstrucción a posteriori. Al contrario, el movimiento mismo quiso expresar muy pronto sus rasgos esenciales en eslóganes concisos que contribuyeron en gran medida a su amplia difusión [ ${ }^{6}$. Este trabajo de conceptualización contó con el apoyo de una iconografía eficaz que circulaba masivamente a través de folletos, tractos y carteles.

9 1) "La salud no se vende". Este eslogan aparentemente muy sencillo permite abordar cuestiones mucho más complejas de lo que uno pensaría. Refiere a una ambigüedad intrínseca del sindicalismo que trata de negociar colectivamente la venta de la fuerza laboral. Retomando una fórmula de Gramsci, pretende alcanzar una legalidad industrial que permita defender, de la manera más eficaz posible, los intereses inmediatos de los trabajadores sin volver a cuestionar la propiedad de los medios de producción y el poder patronal sobre la organización del trabajo [ $\left.{ }^{7}\right]$. La lógica de la negociación colectiva implica una autolimitación de la contestación: el sindicalismo asume compromisos que sus afiliados deben respetar. "La salud no se vende" introduce una ruptura con una larga tradición de monetarización de los riesgos laborales que a menudo se ha afirmado en perjuicio de la acción para la prevención. Las luchas colectivas por un control de los trabajadores sobre sus condiciones de trabajo tienden a volver a cuestionar los compromisos vinculados con la negociación de la venta de la fuerza laboral. Introducen un elemento permanente de contestación en la medida en que socavan el poder de decisión patronal sobre la organización de la producción. Por otra parte, dichas luchas a menudo cuestionan, por su propia dinámica, la división social del trabajo. Efectivamente, contestan los principios de una valoración diferenciada de las calificaciones, la carga de trabajo y los riesgos que justifica las desigualdades salariales. En la mayoría de los casos, estas luchas llevan a destacar categorías poco o mal representadas en las organizaciones sindicales: mujeres, trabajadores no calificados, inmigrantes "del interior" de la Italia de los años 60, etc.

2) "La salud no se delega". Otro eslogan que plantea en pocas palabras varias cuestiones esenciales. Por una parte, critica la prevención prescrita por los especialistas que 
pretenden dictar a los trabajadores las reglas a seguir. Es inseparable de la reivindicación obrera muy presente en numerosas fábricas italianas de aquella época de deshacerse de los médicos de trabajo de las empresas y de crear servicios públicos de salud de trabajo controlados por los trabajadores. La consigna considera que la salud es construida en el marco de estrategias colectivas por el conjunto de las categorías implicadas. Por otra parte, el principio de la no delegación alienta también una contestación interna en las organizaciones sindicales. Considera que el aparato de dichas organizaciones debe someterse a la base y que todas las decisiones estratégicas importantes deben discutirse en asambleas. La fuerza del modelo obrero italiano se basa en gran parte en su capacidad para encarnar en la actualidad inmediata de las luchas un proyecto emancipador para la sociedad. Numerosos testimonios de aquella época mencionan la felicidad de los protagonistas de las luchas en las fábricas. Podemos citar a este antiguo obrero de Fiat: "Mucha gente dice: es duro levantarse a las cinco de la madrugada para ir a la fábrica. Pues sí, es duro, pero para mí entrar por esa puerta por la mañana significaba incorporarme a mi equipo y eso me hacía sentirme vivo, me hacía sentirme yo mismo. Cuando era adolescente me decían que era un rebelde. Pues yo en Fiat estaba a gusto, la gente me entendía. Estaba bien allí dentro. Estaba bien porque estaba rodeado de obreros. Todos mis amigos estaban allí, en la fábrica..." (Revelli, 1989, p. 52). La felicidad de la acción favorece la experiencia colectiva de una liberación siguiendo una lógica de instrumentalización de las luchas en vistas de la negociación (ibídem, p. 61).

11 3) El reconocimiento de la subjetividad obrera concierne tanto los criterios de la verdad como los criterios de lo justo. Por una parte, reconoce la inteligencia colectiva de los trabajadores como peritos en materia de sus condiciones de trabajo, capaces de reconstruir juntos complejos procesos de producción. Dicha capacidad fue ampliamente demostrada por la técnica de cartografía de los riesgos utilizada en miles de empresas distintas. De la misma manera la inteligencia colectiva de los trabajadores sirvió de base para un desarrollo considerable de los conocimientos sobre el impacto negativo del trabajo en términos de salud. Por otra parte, este principio también guarda relación con la validación de las reivindicaciones, de los métodos de intervención y de las transformaciones del trabajo. Afirma que ningún conocimiento experto externo al colectivo de trabajo puede decretar cuáles serían los riesgos aceptables. En el léxico compartido por millones de trabajadores con profesionales comprometidos del mundo de la prevención, se encuentran dos expresiones frecuentes: el "grupo homogéneo" que refiere a un colectivo que comparte las mismas condiciones de trabajo, y la "validación consensual" que implica una deliberación por parte de este colectivo destinada a identificar los problemas y a formular reivindicaciones con el fin de mejorar las condiciones de trabajo. El reconocimiento de los conocimientos de los obreros se articuló con la reivindicación de un derecho a la formación permanente. A partir de 1973, numerosos obreros accedieron a la enseñanza universitaria o a escuelas populares gracias a los convenios colectivos que incluían un derecho a 150 horas de clases pagadas por las empresas. Estas 150 horas podían distribuirse sobre un período de tres años o concentrarse en un solo año siguiendo modalidades variables en función de los convenios colectivos. Este acceso implicaba también una importante renovación de la investigación y la pedagogía. En Turín, estas 150 horas se tradujeron en una clara reorientación de la facultad de medicina hacia el estudio de los determinantes sociales de la salud gracias a la aportación de los obreros de FIAT. Fue en el marco de estas 150 horas que se desarrolló una metodología de encuesta original: la instrucción al sosías. 
Consiste en pedir a un trabajador que explique a un 'semejante' (una persona con las mismas características físicas) lo que debería hacer para realizar su tarea si lo sustituyera. Este método aborda las raíces del taylorismo revelando la inconsistencia de la oposición entre diseñadores e implementadores (Oddone, 2015).

4) La experiencia italiana creó herramientas de trazabilidad lo suficientemente flexibles para adaptarse a situaciones de trabajo muy diferentes. Combinaban un enfoque riguroso desarrollado a base de investigaciones y luchas reivindicativas con un lenguaje accesible gracias al cual resultaba posible confrontar los conocimientos de los colectivos de trabajo y los de los especialistas. La base de semejante cooperación era el compromiso común con una transformación radical de la sociedad italiana. De entre las herramientas cabe mencionar la encuesta obrera y un marco analítico basado en cuatro grupos de "factores de nocividad". La primera encuesta obrera en el marco de un modelo obrero italiano fue lanzada de manera aislada por la Cámara de Trabajo de Turín en 1961. Era el resultado de una iniciativa de militantes obreros, responsables sindicales y especialistas políticamente comprometidos (médicos, químicos, asistentes sociales). La iniciativa partía de la necesidad política de volver a lanzar movilizaciones sindicales sobre una base renovada. La elección por la empresa Farmitalia (una fábrica farmacéutica del grupo Montecatini) se debía a la combinación de tres factores: una fábrica con tecnologías modernas, la percepción por parte de los trabajadores de tasas de riesgo muy elevadas (resumida en la expresión "nosotros conocemos la muerte blanca") y la denegación sistemática por parte de la empresa de esta realidad de la enfermedad y la muerte causadas por las condiciones de trabajo. La encuesta terminó en una lucha extremadamente dura que permitió inventar tácticas nuevas. Paros laborales afectaban los intereses económicos de la empresa concentrándose las acciones en las fases y los lugares vitales del proceso de producción. La encuesta sindical preliminar había permitido definir reivindicaciones capaces de apasionar a los trabajadores. Asimismo, había producido un conocimiento preciso del ciclo productivo que permitía crear una relación de fuerzas a través de una especie de guerrilla de fábrica. Gracias a esta lucha, los convenios colectivos de la química introdujeron criterios totalmente nuevos en aquella época: sustitución sistemática de las sustancias peligrosas cada vez que resultara técnicamente posible y adopción de medidas de prevención adecuadas con presupuestos igual de significativos que para la renovación de los medios de producción. Las movilizaciones del sector químico supusieron una importante ruptura con la tradición de monetarización de los riesgos. En los años siguientes, la experiencia se amplió a otros sectores y otras regiones. A partir de 1964, la Cámara de Trabajo de Turín elaboró una plataforma tipo que permitía orientar las negociaciones colectivas hacia la lucha por la eliminación de los factores nocivos en el trabajo. Un instrumento esencial fue la constitución en 1965 del CRD, el Centro de Investigación y Documentación sobre los Riesgos y Daños en el Trabajo, dirigido por Gastone Marri. Fue fundado a iniciativa de la principal confederación sindical, la CGIL. Se extendió al conjunto del territorio italiano mediante la creación de centros locales que combinaban conocimiento y lucha. A partir de 1974, el CRD se convirtió en un instrumento unitario de las tres confederaciones sindicales italianas [8]. Estas movilizaciones a favor de la salud en el trabajo permitieron el regreso del sindicalismo a los lugares de trabajo en numerosas empresas donde había sido prácticamente eliminado a principios de los años 50. Desempeñaron un papel importante en la acumulación de fuerzas que condujo a partir de 1969 a lo que pasó a llamarse "la primavera caliente". El análisis basado en los cuatro grupos de factores de riesgo 
pretendía permitir a los colectivos de trabajo desarrollar una conciencia colectiva de los atentados contra la salud como resultado de la organización del trabajo. La reagrupación de los factores en un lenguaje accesible pretendía movilizar la experiencia inmediata de los trabajadores. El primer grupo recogía factores comparables con los de los lugares de vida (temperatura, iluminación, ruido, humedad, etc.). El segundo grupo identificaba los riesgos más específicamente industriales (polvo, gas, humo, vapor). El tercer grupo describía los factores de fatiga física, mientras que el cuarto grupo permitía analizar otros factores de fatiga como la monotonía, la ansiedad, la repetitividad, la intensidad del trabajo, etc. Se prestó una atención especial a la trazabilidad de las exposiciones y sus consecuencias a través de los instrumentos de los que los trabajadores podían apropiarse fácilmente: el registro de datos medioambientales (que incluía todas las exposiciones nocivas en una empresa), el registro de datos bioestadísticos sobre los colectivos de trabajo, el carné de salud individual de cada trabajador, y el carné de riesgos de cada trabajador. El desarrollo inicial de estos instrumentos pasó por la ruptura con la tradición de una medicina de trabajo subordinada a la patronal y centrada en la noción de la aptitud individual en vistas de la selección de la mano de obra. En numerosas empresas, los datos se recopilaban desde las asambleas sindicales, a menudo con la ayuda de médicos o estudiantes de medicina que intervenían como militantes. A continuación, dichos instrumentos se integraban en la organización de la prevención por el sistema de salud pública que se desarrolló a partir de la reforma sanitaria de 1978. La elaboración teórica contó con el apoyo, entre 1961 y 1965, de la revista Quaderni rossi publicada a iniciativa de intelectuales marxistas anti-estalinistas. Una de las prioridades de la revista era el desarrollo de una "co-investigación" entre militantes obreros e intelectuales marxistas en la que las encuestas obreras jugaban un papel esencial.

\section{Límites y rebasamientos indispensables para las luchas actuales}

El modelo obrero italiano aportó un marco conceptual cuyo alcance sigue siendo esencial para la lucha actual a favor de la salud en el trabajo. Por consiguiente, conviene prestar atención a los distintos límites surgidos desde los años 60-70 así como a los rebasamientos indispensables debidos a los cambios que se han producido en el mundo laboral. No se trata de repetir nostálgicamente lo que pasó sino más bien de reflexionar sobre la radicalidad de esta experiencia y las posibilidades de reactivarla en condiciones significativamente diferentes.

Las cuestiones de salud vinculadas con la desigualdad entre hombres y mujeres no fueron abordadas sistemáticamente en la mayoría de las movilizaciones en Italia en aquella época. La importante discusión sobre los horarios de las mujeres lanzada a partir de los años 70 por las feministas italianas (Balbo, 1991) no estaba estrechamente vinculada con las movilizaciones a favor de la salud en el trabajo [?]. En la mayoría de las luchas obreras de aquella época, el reconocimiento de una opresión específica de las mujeres no jugó un papel central. Las dos principales batallas contra la dominación patriarcal, la lucha a favor del divorcio y la legalización del aborto [ $\left.{ }^{10}\right]$, a menudo estuvieron presentes como desafíos ajenos al movimiento obrero. En este campo, el partido comunista adoptó una posición de espera y prudente, dictada parcialmente por su apego al compromiso de la constitución republicana de 1948 y reforzada por una 
estrategia electoralista que estimaba que un compromiso feminista le privaría de una parte de su base electoral. Es verdad que pueden observarse algunas excepciones a esta tendencia general. Se crearon "Grupos de medicina para la mujer" en el marco del movimiento "Medicina democrática". Estos grupos se basaban en una cooperación entre mujeres y profesionales de la salud, y se dedicaban al conjunto de los factores determinantes de la salud de las mujeres destacando la reapropiación de su propio cuerpo. Sus intervenciones abordaban tanto los riesgos del trabajo como el derecho a la contracepción y al aborto, y el reparto desigual de las tareas domésticas.

El vínculo entre la lucha por la salud en el trabajo y las movilizaciones medioambientales fue problemático. El reconocimiento general de dicho vínculo ya estaba presente en todas las publicaciones de aquella época y en las reivindicaciones para la reforma del sistema de salud pública. No obstante, en las movilizaciones concretas a nivel de las empresas, este vínculo solo apareció ocasionalmente, y más frecuentemente relacionado con el rechazo a las sustancias tóxicas en el aire o en el agua que con una crítica al impacto global de la producción en sí. En numerosas ocasiones, la lucha medioambiental se percibió como una posible amenaza para el empleo. Caruso, Lai y Surdo (1978) consideran que se produjo una escisión entre la práctica política de los delegados dedicados a la salud en el trabajo en las fábricas y la del sinVasdicato como organismo externo al que se había confiado la intervención en materia de medio ambiente. También en este campo, sin embargo, pueden mencionarse algunas excepciones destacables. En Casale Monferrato (Piamonte), por ejemplo, la lucha por la prohibición del amianto fue llevada a cabo conjuntamente por los trabajadores de la empresa Eternit y la población local. La Cámara de Trabajo desempeñó un papel central al respecto (Rossi, 2012).

Más allá de la integración de perspectivas feministas y ecológicas, surgieron otras necesidades de la nueva realidad del mundo laboral. La precarización del trabajo, el recurso sistemático a las cadenas de subcontratación, así como el creciente papel de la informática hacen que resulte más difícil entender el proceso laboral global. La combinación de experiencias prácticas, movilizaciones y análisis concretos permitirá encontrar nuevos instrumentos metodológicos para responder a estos desafíos.

Las dificultades relacionadas con la reapropiación del modelo obrero italiano en la lucha actual son innegables. Plantear sistemáticamente un vínculo explícito con las cuestiones medioambientales es un elemento clave en la posibilidad de crear nuevas alianzas con las capas comprometidas de científicos y estudiantes y corresponde además a una necesidad real para conseguir objetivos en muchos aspectos de la salud laboral. Las reivindicaciones de democracia en los lugares de trabajo pueden basarse en una crítica amplia de la producción que asocia a las poblaciones locales en su lucha por imponer un control colectivo sobre qué se produce y cómo se hace. Asimismo, es importante demostrar el estrecho vínculo entre las transformaciones de la organización del trabajo y el brutal crecimiento de las desigualdades sociales a escala internacional. Al igual que en la Italia de los años 60 y 70, la capacidad para llevar a cabo una lucha en materia de temas de salud en el trabajo implica transformaciones internas dentro del movimiento sindical. Desarrollar un sindicalismo de red que supere las barreras tradicionales entre los sectores o las empresas, fomentar la democracia interna, hacer circular la experiencia de la lucha - también más allá de las fronteras nacionales - parecen ser desafíos importantes en vistas de dichas transformaciones. En el seno mismo del movimiento sindical, es útil volver a cuestionar una representación 
de la salud y seguridad en el trabajo como una materia delegada a los especialistas. Cualquier lucha seria en este campo cuestionará necesariamente la organización laboral y la división social del trabajo. En ese sentido, adquirirá una dimensión transversal en el enfrentamiento entre capital y trabajo. Quizás sea una de las lecciones más actuales del modelo obrero italiano: no pueden transformarse las condiciones de trabajo sin transformarse al mismo tiempo las prácticas del sindicalismo.

\section{BIBLIOGRAFÍA}

Balbo, L. (ed.). (1991). Tempi di vita. Studi e proposte per cambiarli. Milán: Feltrinelli.

Bruno, A.S., Geerkens, E., Hatzfeld, N., \& Omnès, C. (2011). La santé au travail entre savoirs et pouvoirs (19è - 20è siècles). Rennes: Presses Universitaires de Rennes.

Carnevale, F., \& Moriani, G. (1986). Storia della salute dei lavoratori. Medici medicina del lavoro e prevenzione. Verona: Bertani.

Caruso, A., Lai, P., \& Surdo, A. (1978). L'iniziativa sindacale sui temi della salute a Torino e in Piemonte (1961-78). Quaderni di Rassegna Sindacale, 75, 136-162.

.Corradi, L. (1991). Il tempo rovesciato. Quotidianità femminile e lavoro notturno alla Barilla. Milán: FrancoAngeli.

Galli, P. (1997). Da una parte sola. Autobiografia di un metalmeccanico. Roma: Manifestolibri.

Lorenz, F. (2007). Los zapatos de Carlito. Una historia de los trabajadores navales de Tigre en la década del setenta. Buenos Aires: Norma.

Muniz, H.P., Brito, J., Reis de Souza, K., Athayde, M., \& Lacomblez, M. (2013). Ivar Oddone e sua contribuição para o campo da saúde do trabalhador no Brasil. Revista brasileira de saúde ocupacional, 38(128), 280-291.

Oddone, I., Re. A., \& Briante, G. (2015). Redécouvrir l'expérience du travail (2è ed.). Paris: Les éditions sociales.

Oddone, I. (2007). Experiencia. Laboreal, 3 (1), 52-53. http://laboreal.up.pt/revista/artigo.php? id $=48 \mathrm{u} 560 \mathrm{TV} 6582233: 98597683572$

Pitti, L. (2009). Penarroya 1971-1979. Notre santé n'est pas à vendre! Plein droit, 4(83), 36-40.

Pitti, L. (2010). "Experts “bruts" et médecins critiques. Ou comment la mise en débats des savoirs médicaux a modifié la définition du saturnisme en France durant les années 1970. Politix, 3(91), 103-132.

Polo, G. (1989), I tamburi di Mirafiori. Testimonianze operaie attorno all'autunno caldo alla Fiat. Turín: CRIC editore.

Revelli, M. (1989), Lavorare in Fiat. Da Valletta ad Agnelli a Romiti. Operai, sindacati, robot. Milán: Garzanti.

Righi, M. L. (1992). Le lotte per l'ambiente di lavoro dal dopoguerra ad oggi. Studi Storici, 33, $(2 / 3), 619-652$. 
Rossi, G. (2012). Eternit, la fibre tueuse. Le combat pour la justice de Casale, ville martyre de l'amiante. Paris: La découverte.

Teiger, C., Barbaroux, L., David, M., Duraffourg, J., Galisson, M.-T., Laville, L., \& Thareaut, L. (2006). Quand les ergonomes sont sortis du laboratoire... à propos du travail des femmes dans l'industrie électronique (1963-1973). Rétro-réflexion collective sur l'origine d'une dynamique de coopération entre action syndicale et recherche-formation-action. Pistes, 8(2), 2-39. doi: 10.4000/ pistes.3045.

Teiger, C., \& Lacomblez, M. (ed.) (2013). (Se) former pour transformer le travail. Dynamiques de construction d'une analyse critique du travail, Québec-Bruxelles: Presses de l'Université Laval-ETUI.

Trentin, B. (1980). Il sindacato dei consigli, intervista di Bruno Ugolini. Dieci anni di storia italiana della parte della classe operaia. Roma: Ed. Riuniti.

Vasconcelos, R., \& Lacomblez, M. (2005). Redescubramo-nos na sua experiência: O desafio que nos lança Ivar Oddone. Laboreal, 1 (1), 38-51. http://laboreal.up.pt/revista/artigo.php?

id=37t45nSU547112358941523351.

\section{NOTAS}

1. El DVD en anexo a Teiger y Lacomblez (2013) incluye una rica documentación.

2. La fase intensiva del movimiento se sitúa entre las primeras experiencias lanzadas a partir de 1961 y la derrota de la huelga de las fábricas de FIAT en Turín en 1980. Otras tentativas de periodización consideran que el movimiento conoció un retroceso a partir de 1978 con una reorientación de las confederaciones sindicales a favor de la austeridad y la flexibilidad (a la que en Italia se refiere como "el giro del EUR").

3. La película "Matti da slegare" (Locos de desatar) de Marco Bellocchio (1975) describe los lazos entre algunos jóvenes recién salidos de un hospital psiquiátrico y la fábrica donde acuden a trabajar y donde se integran en la comunidad obrera. Uno de los directores del colectivo que realizó esta película, Silvano Agosti, dedicó posteriormente "D'amore si vive" (1984) a la lucha por una sexualidad libre.

4. La experiencia de Ivar Oddone en una brigada partisana entre 1943 y 1945 inspiró el personaje de Kim en la novela de Italo Calvino "El sendero de los nidos de araña", escrita en 1947.

5. El dibujante Roberto Zamarin creó en las páginas de "Lotta Continua" el personaje de Gasparazzo que encarnaba este "obrero-masa" meridional de FIAT.

6. Laboreal publicó dos artículos que presentan una buena síntesis de aportes importantes del modelo obrero italiano (Oddone, 2007; Vasconcelos \& Lacomblez, 2005)

7. Este análisis fue esbozado en un artículo publicado por Gramsci en "Ordine nuovo" el 12 de junio de 1920 bajo el título "Sindicatos y consejos".

8. El CRD fue disuelto en 1984 al romperse el acuerdo unitario entre las tres principales confederaciones sindicales italianas. Sus archivos digitalizados son la principal fuente histórica del modelo obrero italiano.

9. Este problema queda muy claro en una obra monográfica dedicada a una empresa de la industria alimentaria en Lombardía (Corradi, 1991). Con la extensión del trabajo de noche, la flexibilidad exigida por la patronal terminó reduciendo significativamente el porcentaje de obreras en la fábrica. Las negociaciones sobre las condiciones de trabajo en un contexto de amenaza de cierre de la fábrica sacrificaron el empleo de las mujeres.

10. Este problema queda muy claro en una obra monográfica dedicada a una empresa de la industria alimentaria en Lombardía (Corradi, 1991). Con la extensión del trabajo de noche, la flexibilidad exigida por la patronal terminó reduciendo significativamente el porcentaje de 
obreras en la fábrica. Las negociaciones sobre las condiciones de trabajo en un contexto de amenaza de cierre de la fábrica sacrificaron el empleo de las mujeres.

\section{RESÚMENES}

La denominación "modelo obrero italiano" se refiere a un conjunto de prácticas y análisis que a partir de los años 60 del siglo XX cambiaron profundamente los enfoques tradicionales de la salud y seguridad laboral. Surgido desde movilizaciones en grandes fábricas, dicha experiencia permitió también un cuestionamiento de todas las disciplinas especializadas en salud y seguridad del trabajo. Si el mundo del trabajo ha cambiado de modo significativo, algunos elementos esenciales del modelo obrero italiano siguen dando respuestas válidas a los problemas actuales de salud y seguridad. Un balance crítico ayuda también a identificar aspectos soslayados o limitaciones.

Denomina-se por "modelo operário italiano" um conjunto de práticas e de análises que, a partir dos anos 60 do século XX, transformaram profundamente a abordagem tradicional da saúde no trabalho. Nascido a partir de mobilizações em grandes empresas, esta experiência esteve igualmente na origem de um questionamento das disciplinas especializadas no domínio da saúde e segurança no trabalho. Se o mundo do trabalho mudou significativamente, alguns elementos essenciais do modelo operário italiano configuram ainda um contributo válido nas respostas aos problemas atuais de saúde e segurança no trabalho. Um balanço crítico permite também identificar alguns dos seus aspetos negligenciados ou limitações.

On désigne par modèle ouvrier italien un ensemble de pratiques et d'analyses qui ont profondément modifié l'approche traditionnelle de la santé au travail à partir des années $60 \mathrm{du}$ XXe siècle. Née à partir de mobilisations dans les entreprises, cette expérience a également été à l'origine d'une remise en cause dans l'ensemble des disciplines spécialisées qui interviennent dans le champ de la santé au travail. Si le monde du travail a profondément changé, des éléments essentiels du modèle ouvrier italien permettent d'apporter des réponses aux problèmes actuels de santé au travail. Un bilan critique permet également d'identifier des aspects négligés ou des limites.

The 'Italian workers' model' refers to a set of practices and analysis that has deeply modified the traditional approach of occupational health and safety from the beginning of the 1960s. While it was originated by workers' mobilizations in factories, the experience put into question the different specialized disciplines which intervene in health and safety at work. If the world of work has deeply changed, crucial elements of the Italian workers' model allow us to tackle today challenges in occupational health and safety. A critical balance helps also to identify some limitations or neglected dimensions. 
ÍNDICE

Mots-clés: histoire de la santé et sécurité au travail, participation des travailleurs, syndicalisme, lutte de classes

Palabras claves: historia de la salud y seguridad laboral, participación de los trabajadores, sindicalismo, lucha de clases

Palavras-chave: história da saúde e segurança no trabalho, participação dos trabalhadores, sindicalismo, luta de classes

Keywords: history of health and safety at work; workers" participation; trade unions; class struggle

\section{AUTOR}

\section{LAURENT VOGEL}

Unité 'Conditions de travail, santé et sécurité' - European Trade Union Institute (ETUI) Bd du Roi Albert II, 5, 1210 Brussels, Belgium

lvogel@etui.org 